\title{
Changes in Brain Function Networks in Patients With Amnestic Mild Cognitive Impairment: A Resting-State fMRI Study
}

\author{
Xiaoling $\mathrm{Li}^{1 \dagger}$, Feng Wang ${ }^{1,2 \dagger}$, Xiaohui $\mathrm{Liu}^{2 \dagger}$, Danna Cao ${ }^{1,2}$, Lina Cai ${ }^{2}$, Xiaoxu Jiang ${ }^{2}$, \\ Xu Yang ${ }^{2}$, Tiansong Yang ${ }^{1 *}$ and Tetsuya Asakawa ${ }^{3,4 *}$ \\ ${ }^{1}$ First Affiliated Hospital, Heilongjiang University of Chinese Medicine, Harbin, China, ${ }^{2}$ Division of CT and MRI, Heilongjiang \\ University of Chinese Medicine, Harbin, China, ${ }^{3}$ Department of Neurosurgery, Hamamatsu University School of Medicine, \\ Hamamatsu, Japan, ${ }^{4}$ Research Base of Traditional Chinese Medicine Syndrome, Fujian University of Traditional Chinese \\ Medicine, Fuzhou, China
}

OPEN ACCESS

Edited by:

Görsev Yener,

Dokuz Eylül University, Turkey

Reviewed by:

Radek Marecek,

Central European Institute of Technology (CEITEC), Czechia

Zhiqun Wang,

Aerospace Center Hospital, China

*Correspondence:

Tiansong Yang

Yangtiansong2006@163.com

Tetsuya Asakawa

asakawat1971@gmail.com

${ }^{\dagger}$ These authors have contributed equally to this work

Specialty section:

This article was submitted to Dementia and Neurodegenerative Diseases, a section of the journal Frontiers in Neurology

Received: 21 April 2020 Accepted: 02 September 2020 Published: 30 September 2020

Citation:

Li X, Wang F, Liu X, Cao D, Cai L,

Jiang $X$, Yang $X$, Yang $T$ and Asakawa $T$ (2020) Changes in Brain Function Networks in Patients With Amnestic Mild Cognitive Impairment:

A Resting-State fMRI Study.

Front. Neurol. 11:554032.

doi: 10.3389/fneur.2020.554032
Patients with amnestic mild cognitive impairment $(\mathrm{aMCl})$ are at high risk of developing dementia. This study used resting-state functional magnetic resonance imaging (rs-fMRI) and an independent component analysis (ICA) approach to explore changes in functional connectivity $(\mathrm{FC})$ in the default mode network (DMN), executive control network (ECN), and salience network (SN). Thirty patients with aMCl and 30 healthy controls (HCs) were enrolled. All the participants underwent an rs-fMRI scan. The brain FC in DMN, ECN, and SN was calculated using the ICA approach. We found that the FC of brain regions in DMN decreased significantly and that of brain regions in ECN increased, which was in accordance with the findings of previous studies on Alzheimer's disease (AD) and aMCl. We also found that the FC of brain regions in SN increased, which was different from the findings of previous studies on AD. The increase in FC in brain regions in SN might result from different pathophysiological states in $\mathrm{AD}$ and $\mathrm{aMCl}$, indicating that a decrease in $\mathrm{FC}$ in $\mathrm{SN}$ does not occur in a person with aMCl. These results are consistent with those of previous studies using the voxel-mirrored homotopic connectivity approach and seed-based correlation analysis. We therefore considered that the decrease in FC in DMN and the increase in FC in ECN and SN might be peculiar patterns observed on the rs-fMRI of a person with aMCl. These findings may contribute to the development of imaging biomarkers for the diagnosis of aMCl.

Keywords: resting-state functional magnetic resonance imaging, functional connectivity, default mode network, executive control network, salience network, amnestic mild cognitive impairment

\section{INTRODUCTION}

Mild cognitive impairment (MCI) is a pathological state between normal cognition and dementia. $\mathrm{MCI}$ is associated with many neurological diseases such as neurodegenerative diseases, cerebral vascular diseases, and metabolic symptoms. MCI has slight impact on activities of daily living (ADL), but patients with MCI have a high risk of developing dementia, for which there is no effective treatment. The annual conversion rate from MCI to dementia is reportedly $\sim 10-15 \%$, which is 10 times that in the normal population (1). Thus, MCI has been regarded as a crucial stage in which the long-term outcome of dementia may be improved if early diagnosis and intervention 
are achieved (2). MCI is commonly classified into two subtypes: amnestic MCI (aMCI) and non-amnestic MCI (naMCI). aMCI manifests predominantly as memory loss and is more likely to progress to Alzheimer's disease (AD), whereas naMCI is closely associated with forms of dementia other than AD (3). Because $\mathrm{AD}$ is a leading neurodegenerative disease, the relationship between aMCI and AD and the early diagnosis and treatment of aMCI have garnered much attention from clinicians.

Great efforts have been made to explore diagnostic and screening tools for aMCI, such as psychological batteries $(2,4,5)$, cerebrospinal fluid biomarkers (6), and molecular imaging approaches (7). These methods have their special strengths and limitations. So far, there is no gold standard tool for the early diagnosis of aMCI, but resting-state functional magnetic resonance imaging (rs-fMRI) is often used for this purpose. The functional changes observed on fMRI of patients with $\mathrm{AD}$ have been well-documented. Commonly, resting static networks (long-term networks) are constructed based on function connectivity (FC) on long or full-length fMRI time series. Three large-scale cognition-related networks, the default mode network (DMN), executive control network (ECN), and salience network (SN), are widely used to investigate brain changes in the cognitive impairment state. Joo et al. (8) reported evident abnormal connections in the brains of patients with $\mathrm{AD}$, particularly in the default mode network (DMN), executive control network (ECN), and salience network (SN). DMN is a task-negative as well as the most documented network associated with AD (9). Royall et al. (10) found that DMN atrophy was closely associated with the severity of cognitive impairment in patients with $\mathrm{AD}$. Li et al. (11) found that the disruption of DMN was frequency dependent. Banks et al. (12) found that left lateralization of DMN was associated with an improvement in recall performance in patients with $\mathrm{AD}$. Qi et al. (13) found remarkable damages to the DMN subsystems in patients with $\mathrm{AD}$. Thus, the disruption of $\mathrm{DMN}$ is a characteristic change in $\mathrm{AD}$ that is related to $\mathrm{AD}$ symptoms (14). ECN is a task-positive network that is believed to be relevant to executive dysfunction. Zhao et al. observed changes in FC in patients with AD. They found that FC of the superior frontal gyrus, left thalamus, and middle frontal gyrus in ECN was significantly increased in patients with $\mathrm{AD}$, suggesting the involvement of ECN disruption in $\operatorname{AD}(15,16) . S N$ is a task-positive network that is associated with attention, interoceptive awareness, and affection (17). Zhou et al. (18) reported decreased FC in DMN and increased FC in SN in AD subjects. Agosta et al. (14) reported increased FC in ECN and SN. Importantly, numerous studies have documented the key role of SN under AD conditions; it plays a "switching" role in the modulation of SN and ECN $(17,19-21)$. Damage to SN may affect other networks and eventually cause cognitive decline (21). In addition to these three networks, the frontoparietal network is reportedly involved in $\mathrm{AD}$, in interacts with $\mathrm{DMN}, \mathrm{ECN}$, and $\mathrm{SN}$ $(15,16)$. Finally, changes in and interactions of these networks contribute to cognitive impairment in $\mathrm{AD}$ subjects.

However, there is insufficient evidence supporting the use of rs-fMRI in the diagnosis of aMCI. Hojjati et al. (22) reported a method that integrates rs-fMRI and structural magnetic resonance imaging (MRI) to predict the likelihood of aMCI becoming $\mathrm{AD}$. Badhwar et al. (23) systematically reviewed 34 studies involving 1,363 patients in whom rs-fMRI was used to diagnose $\mathrm{AD}$ and $\mathrm{aMCI}$. They suggested that resting-state connectivity can be considered a potential biomarker of $\mathrm{AD}$. Zhang et al. (24) reported that abnormal FC was discovered in resting-state networks accompanying the progression of aMCI to AD. Such abnormal intra- and internetwork dysfunctions are potential biomarkers of aMCI progression. Recently, Ma et al. (9) have reported a typical disruption of DMN in AD and MCI subjects. However, studies investigating the direct relationship between aMCI and DMN, ECN, and SN are limited. Only two studies have simultaneously investigated abnormalities in all three networks in patients with aMCI. Early in 2012, Liang et al. (25) used seed-based correlation analysis (SCA) to investigate changes in FC in brain regions related to these three subsystems; they found that the FC of the angular gyrus related to DMN was significantly reduced along with an increase in the FC of the left frontal regions within ECN and the FC of some subcortical areas within SN. Later, Liao et al. (26) used the voxel-mirrored homotopic connectivity (VMHC) approach to evaluate the effects of changes in FC on DMN, ECN, and SN in aMCI subjects. On comparing the difference between $\mathrm{AD}$ and aMCI subjects, they determined that DMN inhibition and SN and ECN activation were the characteristic changes in patients with AD and aMCI. Moreover, VMHC was suggested to be a sensitive biomarker of $\mathrm{AD}$ as well as of the progression of aMCI to AD (26). On the basis of these findings, we believe that changes in DMN, ECN, and SN are important features in the progression of aMCI.

Independent component analysis (ICA) is a new method that separates signals of interest from noise in FC analysis (27). In contrast to SCA, ICA does not require a priori selection of a voxel, cluster, and region. It is always used to identify spontaneous activity patterns (27). Despite the strengths and weaknesses of ICA (28), in the present study, we attempted to explore changes in $\mathrm{FC}$ in brain regions attributed to $\mathrm{DMN}, \mathrm{ECN}$, and $\mathrm{SN}$ in patients with aMCI using ICA approach, which is different from SCA and VMHC approaches used in a previous study (28). We wanted to confirm whether changes previously reported in the aMCI state can be reproduced using the ICA approach. We believe that this study will strengthen the evidence for some characteristic changes in patients with aMCI detected using rs-fMRI and provide evidence that rs-fMRI is a useful tool for the early diagnosis of aMCI.

\section{MATERIALS AND METHODS}

\section{Participants}

Patients with aMCI seen in our hospital between June 2018 and October 2019 were enrolled in this study. Healthy volunteers [the healthy control (HC) group] were also recruited. The study was conducted in strict compliance with the guidelines of the Declaration of Helsinki of the World Medical Association (2000) and was approved and supervised by the ethics committee of the First Affiliated Hospital of the Heilongjiang University of Chinese Medicine (approval number: HZYLLBA201910). All participants signed the informed consent form after the study protocol was explained to them in detail. 


\section{Inclusion Criteria for the aMCI and Healthy Control Groups}

We used the diagnostic criteria of the Chinese Dementia and Cognitive Impairment Diagnosis and Treatment Guidelines (2018) (29). Patients meeting the following criteria were included in the aMCI group: (1) those with cognitive impairment, which refers to memory impairment and other cognitive domain damage reported by the patient or the informed person and confirmed via objective examination; (2) those whose basic daily abilities were not affected and whose complex instrumental daily ability was only slightly damaged; (3) those who did not meet the diagnostic criteria for dementia; and (4) those with a geriatric depression scale score of 2-3, a memory test score 1.5 times lower than the standard deviation of the control group matched by age and education, a mini-mental state examination (MMSE) score $\geq 24$, and a Hachinski Ischemic Score (HIS) $\leq 4$. These criteria were consistent with Petersen's criteria (5).

The inclusion criteria for the control group were as follows: (1) no complaints of cognitive impairment; (2) no abnormalities detected on neurological examination and no visual or auditory impairment; (3) MMSE $\geq 27$ points and multidimensional neuropsychological evaluation results within the corresponding normal range; and (4) no infarction or focal lesions on a conventional MRI scan.

The exclusion criteria for all subjects were as follows: (1) a history of nervous system or mental disorder (such as stroke, brain injury, brain tumor, anxiety, and depression); (2) incomplete demographic data; (3) inability to complete all neuropsychological tests; (4) inability to undergo image artifact or MRI examination; and (5) scanning head motion parameters of translation $>2 \mathrm{~mm}$, rotation $>2^{\circ}$.

The cognitive state of all subjects was graded with a battery of neuropsychological assessments including the MMSE, Montreal Cognitive Assessment (MoCA), Trail Making Test (TMT), Digital Span Test (DST), Verbal Fluency Test (VFT), and Auditory Verbal Learning Test (AVLT); these were performed by an experienced neurologist who was blinded to the allocation of subjects and MRI analysis. The ADL scale, Hamilton Depression Rating Scale (HAMD), HIS, Global Deterioration Scale (GDS), and Clinical Dementia Rating (CDR) were also used for assessment.

\section{MRI Acquisition}

A Philips 3.0T MR scanner (Achieva 3.0T-TX with dual gradient multisource emission; Philips Healthcare, the Netherlands) was used in the present study. All participants were asked to arrive $30 \mathrm{~min}$ before their test. They were asked to be fully rested to eliminate psychological factors such as fear, anxiety, and expectation. To reduce possible involuntary head movements caused by respiration and heartbeat, sponge pads were inserted on both sides of the head in the eight-channel parallel acquisition head coil (SENSE-N-8) for fixation.

First, all subjects were scanned using the conventional sequence, including T1WI, T2WI, and fluid-attenuated inversion recovery to exclude subjects who did not meet the inclusion criteria. Then, three-dimensional (3D) structural imaging data acquisition (T1W-3D-TFE sequence) was performed; the scanning parameters were as follows: time of repeat $(\mathrm{TR})=8.3 \mathrm{~ms}$, time of echo $(\mathrm{TE})=3.8 \mathrm{~ms}$, field of view $(\mathrm{FOV})=256 \times 256 \mathrm{~mm}$, flip angle $(\mathrm{FA})=12^{\circ}$, slices $=188$, slice thickness $=1 \mathrm{~mm}$, slice gap $=0 \mathrm{~mm}$, and total scan duration $=4 \min 52 \mathrm{~s}$. Finally, functional imaging data (FEEPI sequence) were collected using the following scanning parameters: $\mathrm{TR}=2000 \mathrm{~ms}, \mathrm{TE}=30 \mathrm{~ms}, \mathrm{FOV}=220 \times 220 \mathrm{~mm}$, $\mathrm{FA}=90^{\circ}$, matrix $=64 \times 64$, slices $=36$, slice thickness $=3 \mathrm{~mm}$, slice gap $=1 \mathrm{~mm}$, total scan duration $=8 \mathrm{~min} 6 \mathrm{~s}$, and a total of 240 time points.

\section{Data Analysis}

Based on a MATLAB platform (R2017a; The MathWorks Inc., Natick, MA, USA), a DPARSF (version 4.3, http://rfmri.org/ DPARSF) software package was run for image preprocessing. This process involved (1) converting data from the Digital Imaging and Communications in Medicine format to the NIfTI format; (2) removing the data of the first 10 time points to avoid the unstable interference of the MRI signal at the beginning of acquisition; (3) slice timing to ensure that all voxel acquisition times in a volume were consistent in theory; (4) realignment, the correction of small head movements between every volume of the subject (if the head translation was more than $2 \mathrm{~mm}$ and the rotation was more than $2^{\circ}$, the data for the subject were excluded; in this study, two patients with aMCI and one patient with normal cognitive function had head movement parameters beyond this range, and they were therefore excluded); (5) normalization, alignment of the local spatial data of different subjects to the Montreal Neurological Institute standard space and their resampling into $3 \times 3 \times 3 \mathrm{~mm}$ voxels; and (6) spatial smoothing, which is performed to reduce the registration error and increase the normality of the data for statistical analysis. This was done with the Gaussian kernel as the full width at half maximum $8 \times 8 \times 8 \mathrm{~mm}$.

\section{Calculation of the Independent Components}

ICA is a data-driven calculation method to separate functional components. It does not need a priori hypothesis, and the functional network components obtained are more realistic (30). Here we used methods introduced in a previous study for data processing (31). Briefly, ICA was performed using GIFT v4.0b software (Group ICA of fMRI toolbox, http:// icatb.sourceforge.net). First, data were imported, the number of components was estimated using the software (30 in this study), and the dimensionality reduction was defined automatically (based on the levels of individuals and groups) using principal component analysis. The infomax algorithm was used. We selected the ICASSO method using both the "RandInit" and the "Bootstrap" options. The number of operations was 100. The minimum cluster size was 80 , and the maximum cluster size was 100 . Then, a spatial-temporal regression was used for the back-reconstruction algorithm, and the spatial components, time series of individuals, and groups were reconstructed. The spatial components were standardized to estimate the Z-scores, and then the average $\mathrm{Z}$-score across the whole cluster was calculated. Based 
on information from a prior study (26) and a template provided by GIFT software, it was decided that DMN, ECN, and SN components would be acquired and analyzed statistically. Finally, for each subject, the chosen DMN, ECN, and SN components were converted to $\mathrm{Z}$-values that represented the FC strength of $\mathrm{DMN}, \mathrm{ECN}$, and SN. Here we used the term "FC" to represent the increase or decrease in the statistical relationship among $\mathrm{DMN}, \mathrm{ECN}$, and $\mathrm{SN}$ in the resting-state networks. However, the analysis was not performed using the functional connectivity analysis approach.

\section{Statistical Analysis}

SPSS software (v 25.0.0., IBM, IL, USA) was used for statistical analyses. Measurement data were presented as the mean \pm standard deviation, and non-normally distributed data were presented as median (quartile). Chi-square test was used for comparing the classified variables between the two groups, and an independent-sample $t$-test was used for measurement data. Spearman rank correlation analyses were run to determine correlation between the $\mathrm{Z}$-values of the functional connection and the MMSE scores of the two groups with significant differences in the three networks. Gender, age, and years of education were used as covariates for regression.

Statistical parametric mapping (SPM)-12 software (http:// www.fil.ion.ucl.ac.uk/spm/) was used for statistical analyses of imaging indicators. In the control and aMCI groups, a singlesample $t$-test (family-wise error [FWE] correction, $P<0.05$ as the test level) was used in the three network templates, and a combined template of the two single-sample brain FC areas was made using xjview (version 9.6, http://www.alivelearn.Net/ xjview) software. Then, SPM12 software was used to run the $t$-test for two groups of the independent samples. The union template was used as the mask. Age, gender, and years of education were used as covariates. The statistical threshold was as follows: voxel level, $P=0.001$ (uncorrected); cluster level, $P<0.05$ (FWE-corrected); and cluster voxel size, $>50$. Thus, a $t$-value map with a significant between-group difference was obtained, and the results were displayed by xjview software.

\section{RESULTS}

Thirty patients with aMCI (13 males and 17 females) and 30 healthy subjects (14 males and 16 females) were enrolled in the study. All the subjects were right-handed. Table 1 shows the clinical characteristics of the subjects. There were no betweengroup differences in age, gender, and years of education. The results of a series of neuropsychological assessments, summarized in Table 1, showed that the aMCI group exhibited a significantly worse performance than the HC group $(P<0.01)$. Regarding clinical assessments, no significant between-group differences were observed in ADL and HIS $(P>0.05)$. However, the aMCI group exhibited higher HAMD, CDR, and GDS scores (indicating a poor performance) than the $\mathrm{HC}$ group $(P<0.01)$. The diagnoses of these patients were confirmed on the basis of the above findings (Table $\mathbf{1}$ ).

In DMN, the FC of the bilateral anterior precuneus (Figure 1A) and right medial frontal gyrus (Figure 1B) was decreased significantly in the patients with aMCI compared with that in the HCs. No significant increase was found in the DMN detected in the present study (Table 2). These results were confirmed via the related analysis. Significant positive correlations were found between the $\mathrm{Z}$-values of the bilateral anterior precuneus and the MMSE scores $(r=0.632 ; P<0.001$; Figure $2 \mathrm{~A}$ ) and between the $\mathrm{Z}$-values of the right medial frontal gyrus and the MMSE scores ( $r=0.472 ; P=0.009$; Figure 2B).

In $\mathrm{ECN}$, the $\mathrm{FC}$ of the right posterior parietal cortex (Figure 1C) was increased significantly in the patients with aMCI compared with that in the HCs. No significant decrease was found in the ECN detected in the present study (Table 2). These results were confirmed via the related analysis. Significant negative correlations were found between the $\mathrm{Z}$-values of the right posterior parietal cortex and the MMSE scores $(r=-0.409$; $P=0.025$; Figure 2C).

In $\mathrm{SN}$, the $\mathrm{FC}$ of the left anterior insula (Figure 1D) was increased significantly in patients with aMCI compared with that in HCs. No significant decrease was found in the SN detected in the present study (Table 2). These results were confirmed via the related analysis. Significant negative correlations were found between the Z-values of the left anterior insula and the MMSE scores $(r=-0.438 ; P=0.015$; Figure 2D).

The anatomical model of DMN, ECN, and SN detected using ICA approach in the present study are summarized in Figure 3.

\section{DISCUSSION}

In the present study, we investigated changes in the cerebral FC in DMN, ECN, and SN in patients with aMCI using rsfMRI. First, we found that the significant decrease in FC in brain regions can be attributed to $\mathrm{DMN}$ and the increase in FC in brain regions can be attributed to ECN; these findings are in accordance with previous studies on AD and MCI. This confirmed the reliability of our experimental system. Second, the most important finding in this study was that the increase in FC in brain regions can be attributed to $\mathrm{SN}$, which is different from the previous findings in patients with AD. These results might reflect the pathophysiological state of aMCI, which requires further confirmation by setting up an $\mathrm{AD}$ group in the future investigation. The results are in agreement with those of previous studies using the SCA (25) and VMHC (26) approaches. We therefore considered that the decrease in FC in DMN and the increase in FC in ECN and SN are peculiar patterns observed on rs-MRI of a person with aMCI. To our knowledge, this is the first report to simultaneously investigate the changes in three core cerebral networks in patients with aMCI using the ICA approach. We believe that these findings will be helpful in developing an imaging biomarker for the diagnosis of aMCI, which is crucial for ameliorating the clinical outcome and improving quality of life (QOL) of patients with dementia.

Our clinical and neuropsychological examination findings confirmed the diagnosis of the patients with aMCI. The MMSE and MoCA scores; TMT and DST results in both directions; and VFT results, GDS and HAMD scores, and AVLT results (immediate, delayed recalls, and recognition) indicated a worse 
TABLE 1 | Clinical characteristics of the enrolled subjects.

\begin{tabular}{|c|c|c|c|c|}
\hline & Items & $\mathrm{HC}$ & aMCI & $P$-value \\
\hline \multirow[t]{4}{*}{ Demographic characteristic } & Number & 30 & 30 & - \\
\hline & Age (y) & $68.67 \pm 3.19$ & $68.53 \pm 2.97$ & 0.868 \\
\hline & Gender (M/F) & $14 / 16$ & $13 / 17$ & 0.795 \\
\hline & Education (y) & $9.93 \pm 2.69$ & $9.53 \pm 2.64$ & 0.563 \\
\hline \multirow[t]{6}{*}{ Neuropsychological assessments } & MMSE scores & $28.20 \pm 0.92$ & $25.10 \pm 0.66$ & $0.000^{\star \star}$ \\
\hline & TMT-B (s) & $137.57 \pm 10.03$ & $180.10 \pm 8.21$ & $0.000^{\star \star}$ \\
\hline & DST-Forward (min) & $5.00(1.00)$ & $4.00(1.00)$ & $0.006^{\star \star}$ \\
\hline & DST-Backword (min) & $6.00(1.00)$ & $4.00(0.75)$ & $0.000^{\star \star}$ \\
\hline & VFT (min) & $20.81 \pm 2.71$ & $18.10 \pm 2.29$ & $0.001^{\star *}$ \\
\hline & AVLT- immediate recall (min) & $6.95 \pm 1.02$ & $6.05 \pm 0.89$ & $0.005^{\star \star}$ \\
\hline \multirow{4}{*}{ Clinical assessments } & HAMD & $5.00(0.00)$ & $6.00(1.00)$ & $0.004^{* \star}$ \\
\hline & HIS & $3.00(1.00)$ & $3.00(2.00)$ & 0.428 \\
\hline & GDS & $1.00(0.00)$ & $3.00(0.75)$ & $0.000^{\star \star}$ \\
\hline & CDR & $0.00(0.00)$ & $0.5(0.00)$ & $0.000^{\star *}$ \\
\hline
\end{tabular}

ADL, Activity of Daily Living Scale; aMCI, amnestic mild cognitive impairment; AVLT, Auditory Verbal Learning Test; CDR, Clinical Dementia Rating; DST, Digital Span Test; GDS, Global Deterioration Scale; HAMD, Hamilton Depression Scale; HC, healthy control; HIS, Hachinski Ischemic Scale; MMSE, Mini-Mental State Examination; MoCA, Montreal Cognitive Assessment; TMT, Trail Making Test; VFT, Verbal Fluency Test.

Date of normal distribution are presented as the mean $\pm S D$, Date of non-normal distribution are presented as the median (quartile), ${ }^{* *}$ means $p<0.01$.

state in the aMCI group. However, there were no between-group differences in ADL and HIS. These findings are consistent with the diagnostic criteria used in the present study (29) as well as with Petersen's criteria (5) (Table 1).

\section{Findings in Accordance With Previous Studies on AD and aMCI}

We found that the FC of the bilateral anterior precuneus and right medial frontalis gyrus, which belong to DMN, was reduced significantly in the aMCI group than in the HCs group (Figure 1B). The subsequent related analyses confirmed these findings. The Z-values of the bilateral anterior precuneus and right medial frontal gyrus were positively correlated with the MMSE scores (Figures 2A,B). Lower MMSE scores were related to lower Z-values, which means that worse cognition was related to lower connectivity. These findings are in agreement with those of the following previous studies. Qi et al. (32) found decreased FC in the hippocampal region, bilateral anterior precuneus, posterior cingulate gyrus, right inferior parietal lobule, and left fusiform gyrus in patients with aMCI. Damoiseaux et al. (33) reported a reduction in FC in the posterior junction of DMN in patients with mild AD, which was detected using ICA. Pappas et al. (34) found that the MMSE scores were correlated with choline acetyltransferase (ChAT) activity in the medial frontal cortex in patients with $\mathrm{AD}$. Ikonomovic et al. (35) reported that lower MMSE scores were related to a reduced precuneus ChAT activity.

DMN is the most basic resting-state network, and it plays a role in integrated primary perception, advanced cognitive function, situational memory extraction, self-consciousness, and cognitive processes. It is also closely related to social cognition, spatial perception, and other brain functions (36). Anatomically, DMN includes mainly the posterior cingulate gyrus, anterior precuneus, medial prefrontal cortex, superior frontalis, middle frontalis, inferior parietal lobule, middle temporal gyrus, infratemporal gyrus, and hippocampus (37). Our findings are in agreement with the results of previous studies on patients with aMCI $(32)$ and $\operatorname{AD}(33,38,39)$; these studies have reported that in these patients, the FC of DMN is decreased, indicating damage to $\mathrm{FC}$ in the state of cognitive impairment.

We found that the FC of the right posterior parietal cortex in ECN was increased in the aMCI group than in the HC group (Figure 1C), which was confirmed via the related analysis, namely, the $\mathrm{Z}$-value of the right posterior parietal cortex was negatively correlated with the MMSE scores (Figure 2C). These findings are in accordance with those of previous analogous studies on patients with aMCI (40) and mild AD (41). ECN includes the dorsolateral prefrontal cortex, cingulate gyrus in the dorsolateral prefrontal cortex, and posterior parietal cortex (42). ECN is involved in multiple high-level cognitive tasks, which are crucial for actively maintaining and manipulating information in working memory, rule-based problem-solving, and goal-based behavior decision-making (43). Our findings suggest that the increase in the right posterior parietal cortex might be a compensation effect of the decline in DMN FC. This indicates an antagonistic and complementary relationship between DMN and ECN, which is consistent with the findings of a previous study (44). These findings replicate those of previous 
A
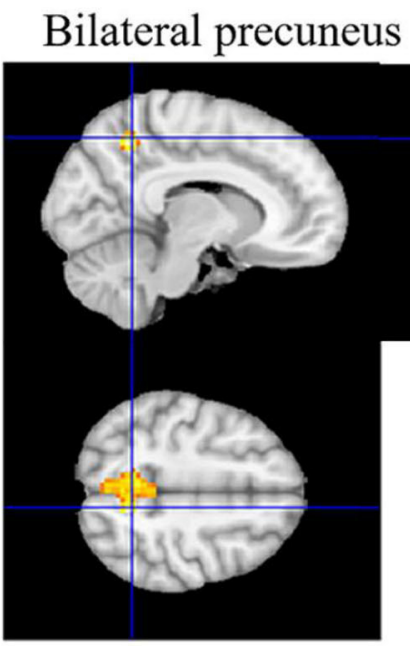

c

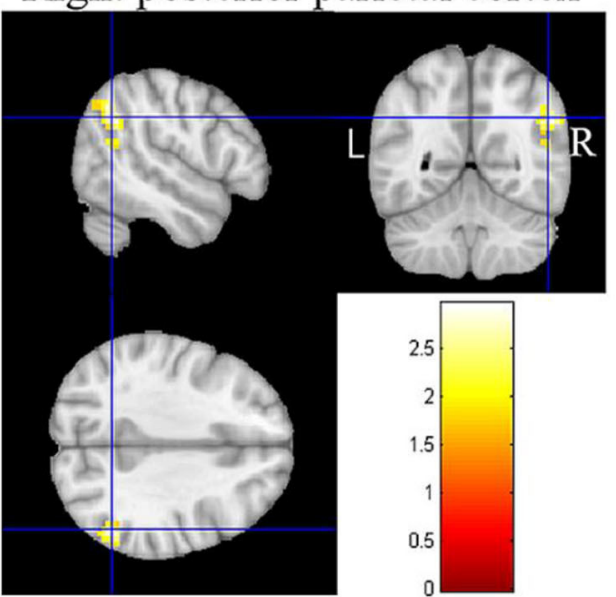

B

\section{Right medial frontal gyrus}

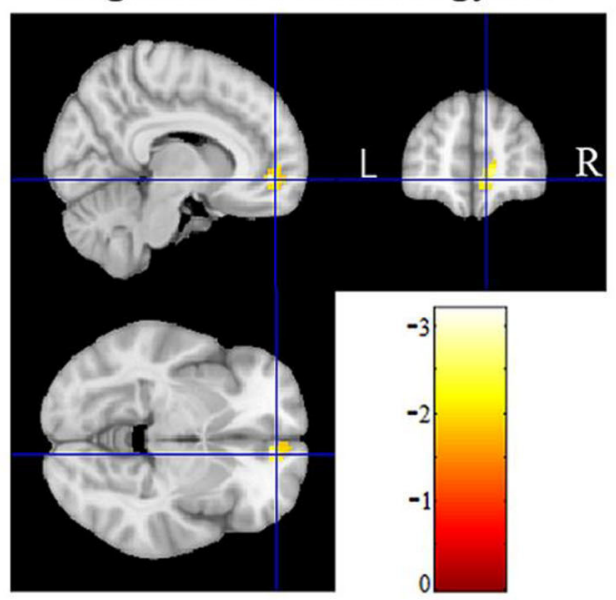

Left anterior insula

D

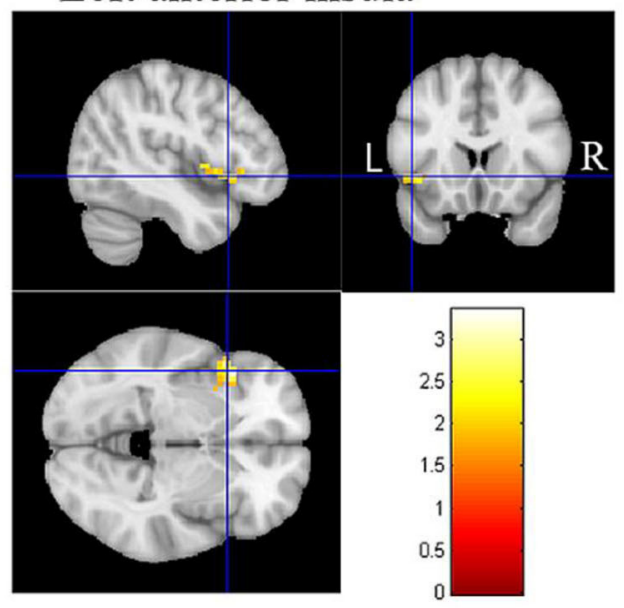

FIGURE 1 | Brain regions of functional connectivity (FC) that were changed in patients with amnestic mild cognitive impairment (aMCl). (A) The FC of the default mode network (DMN) of the bilateral anterior precuneus in patients with aMCl decreased. (B) The FC of DMN of the right medial frontal gyrus in patients with aMCl

decreased. (C) The FC of the executive control network (ECN) of the right posterior parietal cortex in patients with aMCl increased. (D) The FC of the salience network (SN) of the left anterior insula in patients with $\mathrm{aMCl}$ increased.

studies on patients with $\mathrm{AD}$ and, importantly, on patients with aMCI, proving that our experimental design and system were reliable.

\section{New Findings Concerning Changes in FC in Brain Regions Related to aMCI}

Importantly, we found that the FC of the left anterior insula in $\mathrm{SN}$ was increased significantly in the aMCI group than in the HC group (Figure 1D), which was confirmed via the related analysis, namely, the Z-value of the left anterior insula was negatively correlated with the MMSE scores (Figure 2D). These findings are contrary to previous results from patients with AD. Data from patients with $\mathrm{AD}$ indicate that $\mathrm{FC}$ in $\mathrm{SN}$ was decreased, indicating damage to FC in these patients $(18,45)$. There has been no analogous study regarding these changes in patients with aMCI. SN comprises a bilateral forebrain island and an anterior cingulate gyrus. Like ECN, it is an active mission network, participating in brain directional information processing, evaluating and selecting the most relevant environmental stimuli, classifying different stimuli and events, and switching between related processing systems (46). The anterior insula, which is located on the forebrain island, is a key node of $\mathrm{SN}$, which is connected to multiple areas of the prefrontal cortex, central anterolateral cortex, parietal lobe, and temporal lobe (47). It plays an important role in attention and transformation between cognitive domains (48). On the basis of this knowledge, it is easy to understand that in the $\mathrm{AD}$ state, the functional activity of SN is reduced because the brain areas connected to SN are also decreased. However, we found that SN was activated in the aMCI state, which is different from the results found in patients with AD. This might be due to differences between the pathophysiological states of patients with $\mathrm{AD}$ and 
TABLE 2 | Functional connectivity (FC) in the three networks [patients with amnestic mild cognitive impairment (aMCl) vs. healthy controls (HCs)].

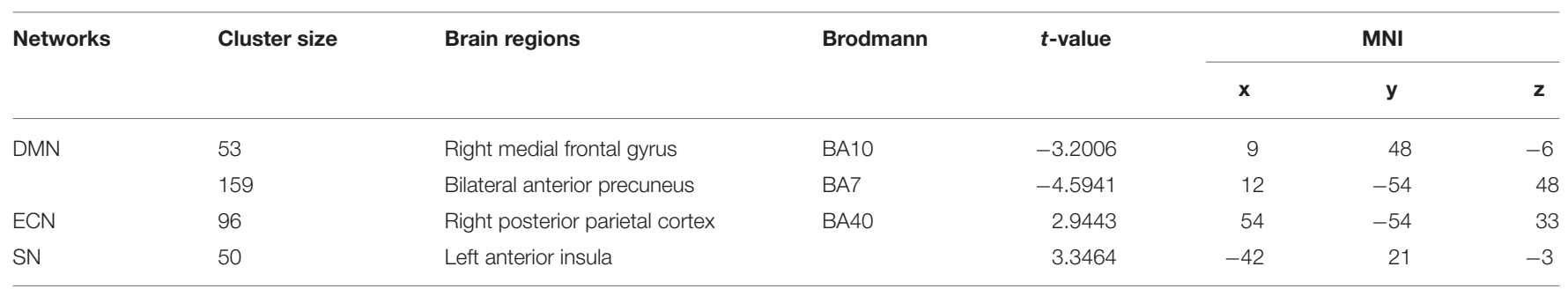

In the default mode network (DMN), $t$-value $=-3.2006$ and -4.5941 , which means that $F C$ in the aMCl group decreased compared with that in the HC group.

In the executive control network $(E C N)$, $t$-value $=2.94443$, which means that $F C$ in the aMCl group increased compared with that in the HC group.

In the salience network (SN), $t$-value $=3.3464$, which means that $F C$ in the $\mathrm{aMCl}$ group increased compared with that in the HC group.

Voxel-level initial threshold, $P \leq 0.001$ (uncorrected).

Cluster-level threshold, $P<0.05$ [family-wise error (FEW) correction].

aMCI. Our data did not support that there was damage to FC in $\mathrm{SN}$ in the aMCI stage. One explanation for this is that the increase in FC in the forebrain island might be due to anxiety and depression in aMCI. Another explanation is that the increase in $\mathrm{FC}$ in the forebrain island is a compensatory effect for the damage to $\mathrm{FC}$ in $\mathrm{DMN}$.

Interestingly, previous studies using the SCA and VMHC approaches have obtained similar results, that is, a decrease in $\mathrm{DMN}$ and an increase in ECN and $\mathrm{SN}$ in patients with aMCI $(25,26)$. We, therefore, believe that changes in DMN, ECN, and $\mathrm{SN}$ comprise a pattern that is peculiar to the resting-state network in patients with aMCI. However, Liao et al. (26) found that patients with $\mathrm{AD}$ also exhibited this pattern. This result is different from those of other studies on $\mathrm{AD}$ that suggested a decrease in SN $(18,45)$. This difference might be associated with a different experimental design (AD vs. aMCI, without normal control) and, in particular, with different VMHC approaches, which require further investigation.

The relationship among these three networks is complicated and multidimensional and is not fully understood. Normal function with flexible interactions among DMN, ECN, and SN plays a vital role in the maintenance of normal social behavior (49). These networks are usually coadjusted according to different physiological states. For example, during performance of a working memory task, the networks changed with the increase in task load to maintain the best performance. FC within DMN was reduced and that within ECN was increased. The difference in FC between $\mathrm{SN}$ and DMN as well as that between $\mathrm{SN}$ and ECN remarkably increased (50). In terms of the association among these networks, the key switching role of $\mathrm{SN}$ in the modulation of DMN and ECN has been well-documented (17, 19-21). The right anterior insula in SN plays a key role in modulating ECN and DMN for better task performance (51). An intact SN drives DMN and ECN during resting and task states, respectively, and contributes to maintaining normal cognitive function in healthy young (52) and old (21) subjects. Nevertheless, the interaction among the three networks in the AD or aMCI state remains to be fully understood. Chand et al. (21) found that patients with MCI exhibited damaged interactions among DMN, ECN, and SN. The SN-centered control model was impaired, resulting in a changed modulation pattern of the networks. Later, Li's study confirmed these changes. Internetwork connectivity, especially SN-DMN and SN-ECN, is increased in patients with $\mathrm{AD}$ and late $\mathrm{MCI}$, suggesting an abnormal state of SN (20). These findings are in agreement with those of the present study. Some authors believe that the changes in FC observed in patients with aMCI are compensatory effects (53). Yi et al. (54) reported that $\mathrm{DMN}$ is the most important network to distinguish between aMCI with and without amyloid-beta $(A \beta)$ protein deposition. A reciprocal variability balance between DMN and SN was observed in aMCI (55). The findings of the present and previous studies imply that atypical activation of $\mathrm{SN}$ and subsequent activation of ECN are the compensatory mechanisms responsible for the decreased function of DMN under MCI conditions, in which SN has to obtain more resources to maintain brain function. This hypothesis based on the compensatory mechanisms of the complicated interactions among SN, DMN, and ECN under the condition of aMCI or AD needs verification in our future investigation.

There are several limitations to this study. First, our study lacked an $\mathrm{AD}$ group; this was the main limitation of our study. We only performed comparisons between patients with aMCI and HCs. From the results of the present study, we cannot obtain direct evidence concerning the difference between $\mathrm{AD}$ and aMCI. Moreover, although we found the depression scores in aMCI group were significantly higher than those of HC group, we don't know the depression state in $\mathrm{AD}$ patients. Thus, the potential confounding role of the depression in the cognitive impairment is uncertain in the present experimental system. Hence, adding patients with $\mathrm{AD}$ to these comparisons would be more meaningful. In the future, we will set up an $\mathrm{AD}$ group to perform a direct comparison that will permit a stronger conclusion. Second, we did not perform more neuropsychological assessments for testing the non-memory domains to demonstrate that there was no nonmemory impairment in the included patients. Although it has been reported that patients with aMCI may suffer from other impairments as well, such as impairment of executive function (56), the included patients might be confused with patients with multiple-domain MCI. This may lead to heterogeneity among the included patients (57). Third, the enrolled patients were diagnosed on the basis of clinical examination findings. We did not confirm the diagnoses using a biomarker for $\mathrm{A} \beta$. Fourth, similar to other approaches, the ICA approach used in this 
A

DMN

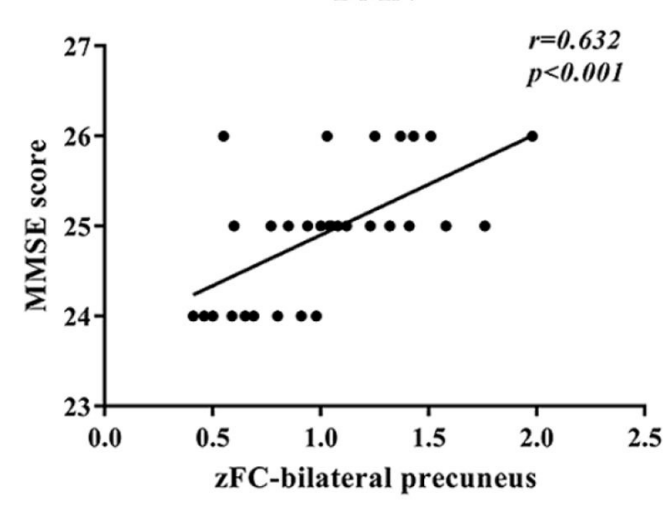

C

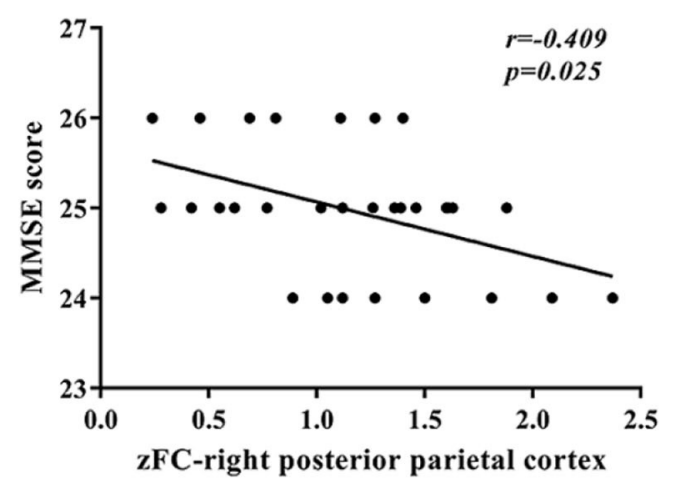

B

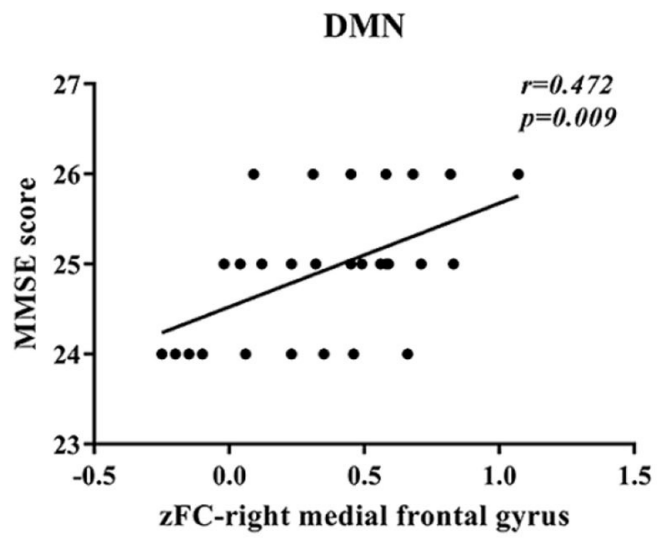

D

SN

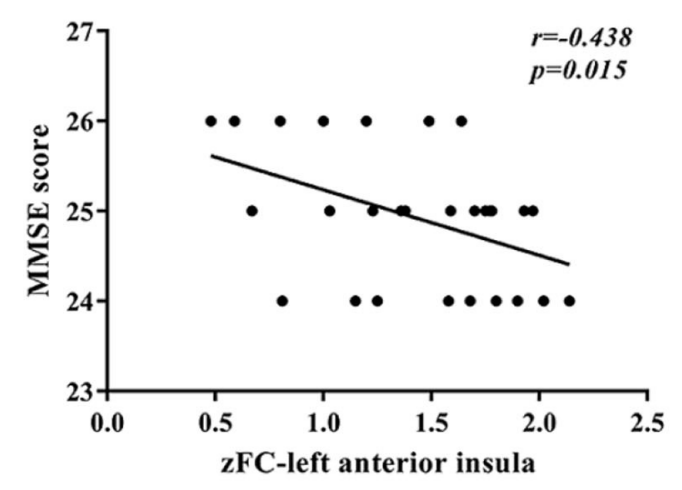

FIGURE 2 | Correlation between the Z-values of brain regions and the mini-mental state examination (MMSE) scores. (A) A significant positive correlation between the Z-value of the default mode network (DMN) of the bilateral anterior precuneus and the MMSE scores was found in patients with amnestic mild cognitive impairment (aMCl). (B) A significant positive correlation between the Z-value of the default mode network (DMN) of the right medial frontal gyrus and the MMSE scores was found in patients with $\mathrm{aMCl}$. (C) A significant negative correlation between the Z-value of the executive control network (ECN) of the right posterior parietal cortex and the MMSE scores was found in patients with aMCl. (D) A significant negative correlation between the Z-value of the salience network (SN) of the left anterior insula and the MMSE scores was found in patients with aMCl.

study has several limitations (28). ICA is a data-driven approach to separate functional components during signal processing. It is based on a blind source separation algorithm and does not need an a priori hypothesis as mentioned before. Hence, it can be used for analysis where a detailed a priori model is not available. However, some challenges remain for the ICA approach: (1) There are no a priori criteria to identify the number of independent components in blood oxygenation leveldependent data, which contribute to the final results to a high degree. (2) There is run-to-run variability; data obtained from different runs of ICA, even on the same data, might be variable. (3) The results of ICA might be split into many subnetworks. Thus, numerous components, which are difficult to identify, would have to be estimated (28). Fifth, these networks are defined by their connectivity, rather than by their constituent structures (10). Although the correlations between the functional networks and the constituent structures are not fully understood, it has been verified that gray matter atrophy may influence the functional results $(10,25,58)$. Thus, gray matter atrophy must be seriously considered in functional imaging studies of neurodegenerative diseases $(25,58)$. However, the present study did not control for gray matter atrophy. Thus, the reported FC changes may have been confused with the effects of gray matter atrophy in the patients with aMCI. All these limitations reduce the reliability of the evidence and should be addressed in our future studies.

Taken together, we found that in the aMCI state, the FC of brain areas in DMN was decreased, whereas that in ECN was increased, which is in agreement with the changes seen in patients with $\mathrm{AD}$. However, $\mathrm{FC}$ in $\mathrm{SN}$ was also increased in patients with aMCI, which was contrary to the findings in patients with $\mathrm{AD}$, indicating that damage to $\mathrm{FC}$ in $\mathrm{SN}$ does not occur in the aMCI stage; this could mean that there are differences in the pathophysiological states between $\mathrm{AD}$ and aMCI. Compensatory response may occur in the aMCI state but seldom in the $\mathrm{AD}$ state (53). Moreover, these results are consistent with those obtained using different analytical approaches. Thus, a decrease in DMN and an increase in ECN and SN may be peculiar patterns observed on rs-fMRI of a person with aMCI and require further investigation. 

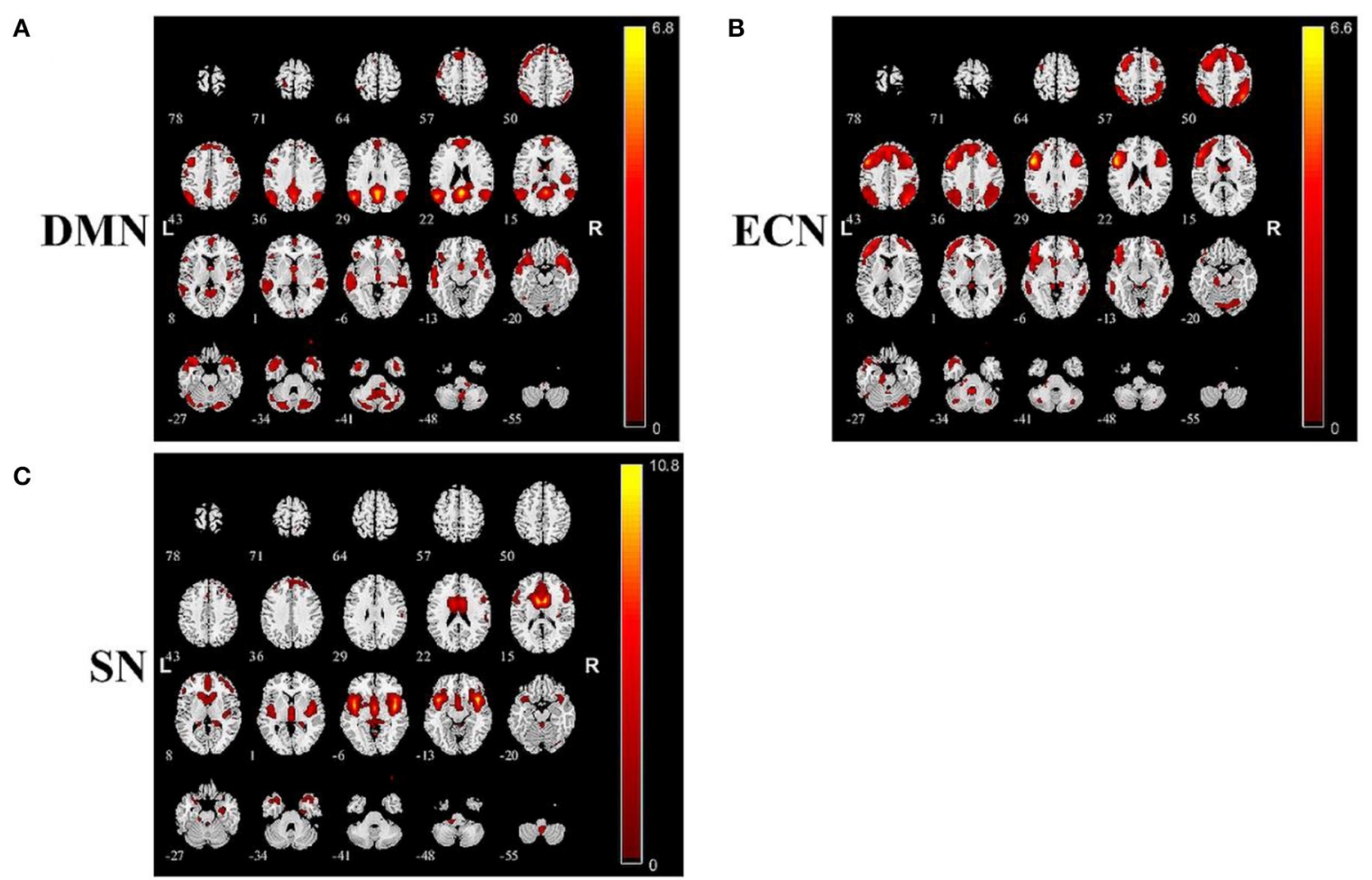

FIGURE 3 | Summary of images of brain function networks detected in this study: (A) default mode network (DMN), (B) executive control network (ECN), and (C) salience network (SN).

\section{CONCLUSION}

The FC of brain regions was decreased in DMN and increased in $\mathrm{ECN}$ and $\mathrm{SN}$, which may be a peculiar pattern observed on rs-fMRI in the aMCI stage. These findings may contribute to developing imaging biomarkers for the diagnosis of aMCI; this would be further beneficial for ameliorating clinical outcomes and improving QOL of patients with dementia.

\section{DATA AVAILABILITY STATEMENT}

The raw data supporting the conclusions of this article will be made available by the authors, without undue reservation.

\section{ETHICS STATEMENT}

The studies involving human participants were reviewed and approved by the ethics committee of the First Affiliated Hospital of the Heilongjiang University of Chinese Medicine (approval no: HZYLLBA201910). The patients/participants provided their written informed consent to participate in this study.

\section{AUTHOR CONTRIBUTIONS}

XL, FW, XL, TY, and TA developed the original idea and designed the approach. XL, FW, XL, DC, LC, XJ, XY, and TY contributed to acquisition, analysis, or interpretation of data for the work. $\mathrm{XL}, \mathrm{FW}, \mathrm{XL}$, and TA wrote the first draft. TY and TA revised the manuscript critically for important intellectual content. TA and TY supervised the study. All authors approval of the final version to be submitted.

\section{FUNDING}

This work was supported in part by the National Foundation of Natural Science of China (Nos. 81973930, 81373714, and 81704170), Natural Science of Heilongjiang Province (No. H2016081), the Special Fund of Harbin Science and Technology Innovation Talents Research (Nos. 2016RAXYJ096, and 2017RAQXJ180), Chunhui plan of the Ministry of Education (No. Z2009-1-15030), Research Fund Project of Heilongjiang University of Chinese Medicine (No. 201704). This work was also supported by grants from the Japan Society for the Promotion of Science (Grant-in-Aid for Young Scientists, Type B, No. 20791025 and Grant-in-Aid for Scientific Research C, General, Nos. 24592157, 15k10358, and 18K08991).

\section{ACKNOWLEDGMENTS}

The authors would like to thank Enago (www.enago.jp) for the English language review. 


\section{REFERENCES}

1. Gyebnar G, Szabo A, Siraly E, Fodor Z, Sakovics A, Salacz P, et al. What can DTI tell about early cognitive impairment? - Differentiation between MCI subtypes and healthy controls by diffusion tensor imaging. Psychiatry Res Neuroimaging. (2018) 272:46-57. doi: 10.1016/j.pscychresns.2017.10.007

2. Zhuang L, Yang Y, Gao J. Cognitive assessment tools for mild cognitive impairment screening. J Neurol. (2019). doi: 10.1007/s00415-019-09506-7. [Epub ahead of print].

3. Csukly G, Siraly E, Fodor Z, Horvath A, Salacz P, Hidasi Z, et al. The differentiation of amnestic type MCI from the nonamnestic types by structural MRI. Front Aging Neurosci. (2016) 8:52. doi: 10.3389/fnagi.2016.00052

4. Glynn K, Coen R, Lawlor BA. Is the quick mild cognitive impairment screen (QMCI) more accurate at detecting mild cognitive impairment than existing short cognitive screening tests? A systematic review of the current literature. Int J Geriatr Psychiatry. (2019) 34:1739-46. doi: 10.1002/gps.5201

5. Petersen RC. Mild cognitive impairment as a diagnostic entity. J Intern Med. (2004) 256:183-94. doi: 10.1111/j.1365-2796.2004.01388.x

6. Giau VV, Bagyinszky E, An SSA. Potential fluid biomarkers for the diagnosis of mild cognitive impairment. Int J Mol Sci. (2019) 20:4149. doi: 10.3390/ijms20174149

7. Chandra A, Valkimadi PE, Pagano G, Cousins O, Dervenoulas G, Politis M, et al. Applications of amyloid, tau, and neuroinflammation PET imaging to alzheimer's disease and mild cognitive impairment. Hum Brain Mapp. (2019) 40:5424-42. doi: 10.1002/hbm.24782

8. Joo SH, Lim HK, Lee CU. Three large-scale functional brain networks from resting-state functional MRI in subjects with different levels of cognitive impairment. Psychiatry Investig. (2016) 13:1-7. doi: 10.4306/pi.2016.13.1.1

9. Ma X, Zhuo Z, Wei L, Ma Z, Li Z, Li H, et al. Altered temporal organization of brief spontaneous brain activities in patients with alzheimer's disease. Neuroscience. (2020) 425:1-11. doi: 10.1016/j.neuroscience.2019. 11.025

10. Royall DR, Palmer RF, Vidoni ED, Honea RA, Burns JM. The default mode network and related right hemisphere structures may be the key substrates of dementia. J Alzheimers Dis. (2012) 32:467-78. doi: 10.3233/JAD-2012-120424

11. Li Y, Yao H, Lin P, Zheng L, Li C, Zhou B, et al. Frequency-dependent altered functional connections of default mode network in alzheimer's disease. Front Aging Neurosci. (2017) 9:259. doi: 10.3389/fnagi.2017.00259

12. Banks SJ, Zhuang X, Bayram E, Bird C, Cordes D, Caldwell JZK, et al. Default mode network lateralization and memory in healthy aging and alzheimer's disease. J Alzheimers Dis. (2018) 66:1223-34. doi: 10.3233/JAD-180541

13. Qi H, Liu H, Hu H, He H, Zhao X. Primary disruption of the memoryrelated subsystems of the default mode network in alzheimer's disease: restingstate functional connectivity MRI study. Front Aging Neurosci. (2018) 10:344. doi: $10.3389 /$ fnagi.2018.00344

14. Agosta F, Pievani M, Geroldi C, Copetti M, Frisoni GB, Filippi M. Resting state fMRI in alzheimer's disease: beyond the default mode network. Neurobiol Aging. (2012) 33:1564-78. doi: 10.1016/j.neurobiolaging.2011.06.007

15. Zhao Q, Lu H, Metmer H, Li WXY, Lu J. Evaluating functional connectivity of executive control network and frontoparietal network in alzheimer's disease. Brain Res. (2018) 1678:262-72. doi: 10.1016/j.brainres.2017.10.025

16. Zhao Q, Sang X, Metmer H, Swati Z, Lu J, Alzheimer's Disease NeuroImaging I. Functional segregation of executive control network and frontoparietal network in alzheimer's disease. Cortex. (2019) 120:36-48. doi: 10.1016/j.cortex.2019.04.026

17. Sridharan D, Levitin DJ, Menon V. A critical role for the right fronto-insular cortex in switching between central-executive and default-mode networks. Proc Natl Acad Sci USA. (2008) 105:12569-74. doi: 10.1073/pnas.0800005105

18. Zhou J, Greicius MD, Gennatas ED, Growdon ME, Jang JY, Rabinovici GD, et al. Divergent network connectivity changes in behavioural variant frontotemporal dementia and Alzheimer's disease. Brain. (2010) 133(Pt 5):1352-67. doi: 10.1093/brain/awq075

19. Uddin LQ. Salience processing and insular cortical function and dysfunction. Nat Rev Neurosci. (2015) 16:55-61. doi: 10.1038/nrn3857

20. Li C, Li Y, Zheng L, Zhu X, Shao B, Fan G, et al. Abnormal brain network connectivity in a triple-network model of alzheimer's disease. J Alzheimers Dis. (2019) 69:237-52. doi: 10.3233/JAD-181097

21. Chand GB, Wu J, Hajjar I, Qiu D. Interactions of the salience network and its subsystems with the default-mode and the central-executive networks in normal aging and mild cognitive impairment. Brain Connect. (2017) 7:401-12. doi: 10.1089/brain.2017.0509

22. Hojjati SH, Ebrahimzadeh A, Khazaee A, Babajani-Feremi A, Alzheimer's Disease Neuroimaging Initiative. Predicting conversion from MCI to AD by integrating rs-fMRI and structural MRI. Comput Biol Med. (2018) 102:30-9. doi: 10.1016/j.compbiomed.2018.09.004

23. Badhwar A, Tam A, Dansereau C, Orban P, Hoffstaedter F, Bellec P. Resting-state network dysfunction in alzheimer's disease: a systematic review and meta-analysis. Alzheimers Dement. (2017) 8:73-85. doi: 10.1016/j.dadm.2017.03.007

24. Zhang Y, Liu X, Zhao K, Li L, Ding Y. Study of altered functional connectivity in individuals at risk for alzheimer's disease. Technol Health Care. (2018) 26:103-11. doi: 10.3233/THC-174235

25. Liang P, Wang Z, Yang Y, Li K. Three subsystems of the inferior parietal cortex are differently affected in mild cognitive impairment. J Alzheimers Dis. (2012) 30:475-87. doi: 10.3233/JAD-2012-111721

26. Liao ZL, Tan YF, Qiu YJ, Zhu JP, Chen Y, Lin SS, et al. Interhemispheric functional connectivity for alzheimer's disease and amnestic mild cognitive impairment based on the triple network model. J Zhejiang Univ Sci B. (2018) 19:924-34. doi: 10.1631/jzus.B1800381

27. Boly M, Phillips C, Tshibanda L, Vanhaudenhuyse A, Schabus M, Dang$\mathrm{Vu} \mathrm{TT}$, et al. Intrinsic brain activity in altered states of consciousness: how conscious is the default mode of brain function? Ann N Y Acad Sci. (2008) 1129:119-29. doi: 10.1196/annals.1417.015

28. Cole DM, Smith SM, Beckmann CF. Advances and pitfalls in the analysis and interpretation of resting-state FMRI data. Front Syst Neurosci. (2010) 4:8. doi: $10.3389 /$ fnsys. 2010.00008

29. Writing Goup of the Dementia, Cognitive Society of Neurology Committee of Chinese Medical Association. [Guidelines for dementia and cognitive impairment in China: the diagnosis and treatment of mild cognitive impairment]. Natl Med J China. (2018) 98:1294-301. doi: 10.3760/cma.j.issn.0376-2491.2018.17.003

30. Segawa K, Azuma H, Sato K, Yasuda T, Arahata K, Otsuki K, et al. Regional cerebral blood flow changes in depression after electroconvulsive therapy. Psychiatry Res. (2006) 147:135-43. doi: 10.1016/j.pscychresns.2004.08.006

31. Assaf M, Jagannathan K, Calhoun VD, Miller L, Stevens MC, Sahl R, et al. Abnormal functional connectivity of default mode sub-networks in autism spectrum disorder patients. Neuroimage. (2010) 53:247-56. doi: 10.1016/j.neuroimage.2010.05.067

32. Qi Z, Wu X, Wang Z, Zhang N, Dong H, Yao L, et al. Impairment and compensation coexist in amnestic MCI default mode network. Neuroimage. (2010) 50:48-55. doi: 10.1016/j.neuroimage.2009.12.025

33. Damoiseaux JS, Prater KE, Miller BL, Greicius MD. Functional connectivity tracks clinical deterioration in alzheimer's disease. Neurobiol Aging. (2012) 33:828.e19-30. doi: 10.1016/j.neurobiolaging.2011.06.024

34. Pappas BA, Bayley PJ, Bui BK, Hansen LA, Thal LJ. Choline acetyltransferase activity and cognitive domain scores of alzheimer's patients. Neurobiol Aging. (2000) 21:11-7. doi: 10.1016/S0197-4580(00)00090-7

35. Ikonomovic MD, Klunk WE, Abrahamson EE, Wuu J, Mathis CA, Scheff SW, et al. Precuneus amyloid burden is associated with reduced cholinergic activity in alzheimer disease. Neurology. (2011) 77:39-47. doi: 10.1212/WNL.0b013e31822 31419

36. Mantini D, Vanduffel W. Emerging roles of the brain's default network. Neuroscientist. (2013) 19:76-87. doi: 10.1177/1073858412446202

37. Buckner RL, Andrews-Hanna JR, Schacter DL. The brain's default network: anatomy, function, and relevance to disease. Ann N Y Acad Sci. (2008) 1124:1-38. doi: 10.1196/annals.1440.011

38. Brier MR, Thomas JB, Ances BM. Network dysfunction in alzheimer's disease: refining the disconnection hypothesis. Brain Connect. (2014) 4:299-311. doi: 10.1089/brain.2014.0236

39. Greicius MD, Kimmel DL. Neuroimaging insights into networkbased neurodegeneration. Curr Opin Neurol. (2012) 25:727-34. doi: 10.1097/WCO.0b013e32835a26b3

40. Sorg C, Riedl V, Muhlau M, Calhoun VD, Eichele T, Laer L, et al. Selective changes of resting-state networks in individuals at risk for alzheimer's disease. Proc Natl Acad Sci USA. (2007) 104:18760-5. doi: 10.1073/pnas.07088 03104

41. Balachandar R, John JP, Saini J, Kumar KJ, Joshi H, Sadanand S, et al. A study of structural and functional connectivity in early alzheimer's disease 
using rest fMRI and diffusion tensor imaging. Int J Geriatr Psychiatry. (2015) 30:497-504. doi: 10.1002/gps.4168

42. Damoiseaux JS, Rombouts SA, Barkhof F, Scheltens P, Stam CJ, Smith SM, et al. Consistent resting-state networks across healthy subjects. Proc Natl Acad Sci USA. (2006) 103:13848-53. doi: 10.1073/pnas.0601417103

43. Li Q, Liu J, Wang W, Wang Y, Li W, Chen J, et al. Disrupted coupling of large-scale networks is associated with relapse behaviour in heroin-dependent men. J Psychiatry Neurosci. (2018) 43:48-57. doi: 10.1503/jpn.170011

44. Chen H, Li Y, Liu Q, Shi Q, Wang J, Shen H, et al. Abnormal interactions of the salience network, central executive network, and default-mode network in patients with different cognitive impairment loads caused by leukoaraiosis. Front Neural Circuits. (2019) 13:42. doi: 10.3389/fncir.2019.00042

45. He X, Qin W, Liu Y, Zhang X, Duan Y, Song J, et al. Age-related decrease in functional connectivity of the right fronto-insular cortex with the central executive and default-mode networks in adults from young to middle age. Neurosci Lett. (2013) 544:74-9. doi: 10.1016/j.neulet.2013.03.044

46. Menon V, Uddin LQ. Saliency, switching, attention and control: a network model of insula function. Brain Struct Funct. (2010) 214:655-67. doi: 10.1007/s00429-010-0262-0

47. Cerliani L, Thomas RM, Jbabdi S, Siero JC, Nanetti L, Crippa A, et al. Probabilistic tractography recovers a rostrocaudal trajectory of connectivity variability in the human insular cortex. Hum Brain Mapp. (2012) 33:2005-34. doi: $10.1002 / \mathrm{hbm} .21338$

48. Touroutoglou A, Hollenbeck M, Dickerson BC, Feldman Barrett L. Dissociable large-scale networks anchored in the right anterior insula subserve affective experience and attention. Neuroimage. (2012) 60:1947-58. doi: 10.1016/j.neuroimage.2012.02.012

49. Smith R, Alkozei A, Killgore WDS. Contributions of self-report and performance-based individual differences measures of social cognitive ability to large-scale neural network functioning. Brain Imaging Behav. (2017) 11:685-97. doi: 10.1007/s11682-016-9545-2

50. Liang X, Zou Q, He Y, Yang Y. Topologically reorganized connectivity architecture of default-mode, executive-control, and salience networks across working memory task loads. Cereb Cortex. (2016) 26:1501-11. doi: 10.1093/cercor/bhu316

51. Li C, Dong M, Yin Y, Hua K, Fu S, Jiang G. Aberrant effective connectivity of the right anterior insula in primary insomnia. Front Neurol. (2018) 9:317. doi: 10.3389/fneur.2018.00317
52. Chand GB, Dhamala M. Interactions among the brain default-mode, salience, and central-executive networks during perceptual decision-making of moving dots. Brain Connect. (2016) 6:249-54. doi: 10.1089/brain.2015.0379

53. Yu E, Liao Z, Tan Y, Qiu Y, Zhu J, Han Z, et al. High-sensitivity neuroimaging biomarkers for the identification of amnestic mild cognitive impairment based on resting-state fMRI and a triple network model. Brain Imaging Behav. (2019) 13:1-14. doi: 10.1007/s11682-017-9727-6

54. Yi D, Choe YM, Byun MS, Sohn BK, Seo EH, Han J, et al. Differences in functional brain connectivity alterations associated with cerebral amyloid deposition in amnestic mild cognitive impairment. Front Aging Neurosci. (2015) 7:15. doi: 10.3389/fnagi.2015.00015

55. Zhang L, Zuo XN, Ng KK, Chong JSX, Shim HY, Ong MQW, et al. Distinct BOLD variability changes in the default mode and salience networks in alzheimer's disease spectrum and associations with cognitive decline. Sci Rep. (2020) 10:6457. doi: 10.1038/s41598-020-63540-4

56. Xie C, Bai F, Yu H, Shi Y, Yuan Y, Chen G, et al. Abnormal insula functional network is associated with episodic memory decline in amnestic mild cognitive impairment. Neuroimage. (2012) 63:320-7. doi: 10.1016/j.neuroimage.2012.06.062

57. Voisin T, Touchon J, Vellas B. Mild cognitive impairment: a nosological entity? Curr Opin Neurol. (2003) 16(Suppl. 2):S43-5. doi: 10.1097/00019052-200312002-00008

58. He Y, Wang L, Zang Y, Tian L, Zhang X, Li K, et al. Regional coherence changes in the early stages of alzheimer's disease: a combined structural and resting-state functional MRI study. Neuroimage. (2007) 35:488-500. doi: 10.1016/j.neuroimage.2006.11.042

Conflict of Interest: The authors declare that the research was conducted in the absence of any commercial or financial relationships that could be construed as a potential conflict of interest.

Copyright (C) 2020 Li, Wang, Liu, Cao, Cai, Jiang, Yang, Yang and Asakawa. This is an open-access article distributed under the terms of the Creative Commons Attribution License (CC BY). The use, distribution or reproduction in other forums is permitted, provided the original author(s) and the copyright owner(s) are credited and that the original publication in this journal is cited, in accordance with accepted academic practice. No use, distribution or reproduction is permitted which does not comply with these terms. 